\title{
Systematic Review of the Development of Spatial Intelligence through Augmented Reality in STEM Knowledge Areas
}

\author{
Francisco del Cerro Velázquez (D) and Ginés Morales Méndez *D \\ Energy Engineering and Teaching Innovation Research Group in Technology, Faculty of Education

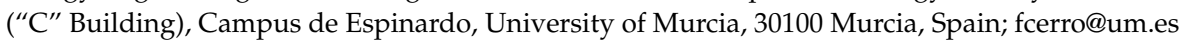 \\ * Correspondence: gines.morales@um.es; Tel.: +34-868-887-696
}

Citation: del Cerro Velázquez, F.;

Morales Méndez, G. Systematic

Review of the Development of Spatial Intelligence through Augmented Reality in STEM Knowledge Areas. Mathematics 2021, 9, 3067. https:// doi.org/10.3390/math9233067

Academic Editor: Jay Jahangiri

Received: 1 November 2021

Accepted: 24 November 2021

Published: 28 November 2021

Publisher's Note: MDPI stays neutral with regard to jurisdictional claims in published maps and institutional affiliations.

Copyright: (c) 2021 by the authors. Licensee MDPI, Basel, Switzerland. This article is an open access article distributed under the terms and conditions of the Creative Commons Attribution (CC BY) license (https:// creativecommons.org/licenses/by/ $4.0 /)$.

\begin{abstract}
This study presents a systematic review (SR) of the literature on the use of Augmented Reality (AR) for the development of spatial skills of secondary and higher education students in the teaching of subjects related to the area of Science, Technology, Engineering and Mathematics (STEM). Numerous researchers have confirmed that students tend to have difficulties in acquiring learning content with a high visual load, which is why AR is being used to improve teaching-learning processes. This paper aims to present information of interest to researchers, teachers and software developers about the advantages and limitations of AR in the development of spatial intelligence, the integration and development of AR applications, as well as the predominant spatial skills and assessment methods. In this regard, an initial search was carried out which returned 242 articles. After reviewing and assessing their eligibility, a total of 17 papers published since 2002 were selected. The findings reveal an increase in the number of investigations over the last few years. Some of the most notable findings are the improvement of spatial skills of students through the inclusion of $\mathrm{AR}$ in educational environments, the need for teacher training, the lack of personalization in the applications developed and the scarcity of augmented materials in the form of Open Educational Resources (OER).
\end{abstract}

Keywords: augmented reality; spatial intelligence; STEM; education; systematic review

\section{Introduction}

For the improvement of the teaching and learning process of science, technology, engineering and mathematics - a group of disciplines known as STEM subjects - the development of students' spatial skills is essential, especially when it comes to the analysis or study of contents with a high visual load [1-3]. In this sense, within the educational contents of these STEM subjects, spatial skills enable students in: the conception and construction of spatial models, the analysis of geometric objects, the interpretation of diagrams and the identification of functions among others [4].

Spatial intelligence can be defined as the ability to perceive, generate, retain, retrieve and transform figures, images or objects in a structured way, even in the absence of physical stimuli. The term spatial intelligence encompasses five fundamental domains of skills that emphasize different aspects of the cognitive process of generating, storing, retrieving and transforming different types of representation: spatial visualization, mental rotation, spatial perception, spatial relatedness and spatial orientation [5].

These five skills are malleable and can therefore be enhanced through the use of multisensory tools or applications that stimulate and enhance the above skills [6]. However, the traditional method for teaching visual and spatial skills to students is based on analyzing and interpreting two-dimensional images, orthogonal views and graphs on a blackboard or on paper. This method has obvious limitations, as it hinders the conceptualization and assimilation of content, due to the lack of interaction between students and the representations [7]. 
AR technology provides an interface that offers multiple benefits that support visuospatial cognitive relationships and transformations. This process is based on the superimposition of virtual information on a real scenario. In terms of the requirements for a system to be considered AR, Azuma [8] states that it must combine real and virtual objects in a real environment, operate interactively and in real time, and link virtual objects by superimposing them on real markers or triggers.

Similarly, the use of AR can help students make information connections between the virtual and the real more easily, making the learning of abstract two-dimensional or threedimensional content more interactive [9]. Learners can proactively inspect augmented materials from any perspective, thus making learning digitally tangible and sensory, and enhancing cognitive comprehension processes [7].

In recent years, AR has acquired a greater prominence, due to its technological development. There is a large body of scientific work revealing the advantages, limitations and upcoming challenges of AR in learning, and specifically in STEM education [10-21]. Among the findings there is a strong convergence, where the authors frequently highlight that AR facilitates learning, increases student interest, enhances self-learning and is easy to use. However, they also point out technological dependence and the possible distractions that it can cause in students. While much research has focused on the incorporation of AR technology in educational settings, few studies have focused on the potential impact of AR on students' spatial intelligence [22-29].

Considering this background, the present SR proposes: (1) Collecting, synthesizing and integrating the existing literature that measures the impact of AR on students' spatial intelligence. (2) Identifying how some of the moderating variables influence the impact of AR on the possible improvement of students' spatial skills. All this, with the purpose of identifying how AR systems influence students' spatial intelligence in order to guide the development of future research, methodologies or applications in the teaching of STEM subjects.

\section{Theoretical Framework}

Several studies [1,29] claim that visual and spatial skills can be enhanced by emerging technologies such as AR. The integration of this technology in the classroom favors a constructivist approach to learning by allowing teachers to bring tangible and proactive experiences into the classroom where students interact and manipulate the learning object. As educators, we must show a positive attitude towards the integration of ICT in education, as it effectively changes the way students learn [30]; however, there is still much work to be done in order to achieve a systematic development of AR for educational purposes.

A recent systematic literature review by Ahmad and Junaini [31] determined that the use of AR in mathematics teaching should be aimed at enhancing the teaching-learning process through the development of spatial visualization and problem-solving skills [32]. Their results show that there is an absence in teacher training programs and augmented materials, which requires further research in this field. The paper reviewed 19 scientific articles, however, without focusing on a detailed description of the impact of AR on students' spatial skills.

While a large number of previous reviews of AR applications have focused on the field of education [33], there is an absence of systematic literature reviews on the use of AR in spatial skills training. According to Gün and Atasoy [34], very little action or systematic research work has been conducted to study the effects of AR systems in spatial skills training; moreover, software for developing AR applications or augmented materials is scarce and limited. Therefore, a review of research studies on the development of spatial skills using AR technology can shed light and guide future research in education.

\section{Methodology}

The present study followed a rigorous research process in order to collect, evaluate and summarize the empirical evidence related to AR and its impact on students' spatial 
intelligence. Seventeen quantitative studies from major databases that measured the impact of AR on students' spatial intelligence were selected for analysis.

This work was conducted according to the guidelines proposed by Kitchenham [35] for literary SR and adapted to our research framework. This model was initially used for reviews in the fields of medicine and computer science. In this case, the review focused on the analysis of scientific articles, and following the steps of SR of the literature; three fundamental phases were articulated: planning, development and results of the review (Figure 1).

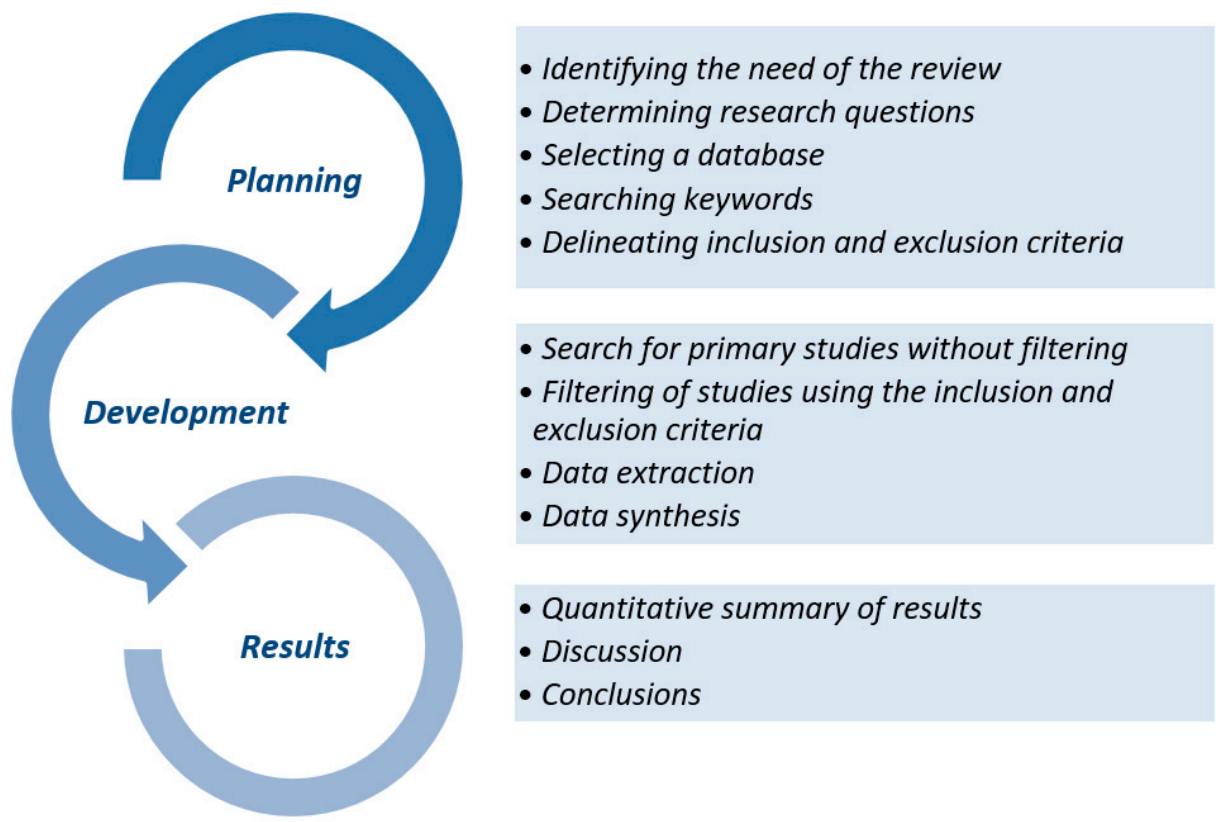

Figure 1. SR phase diagram.

In the first phase of planning, a review procedure is developed that serves as a guide for the review and specifies the objectives, methods and main outcomes of interest of the SR. In this phase, the keywords, inclusion-exclusion criteria and data to be analyzed are defined.

In the development phase, the findings found in the studies are related to the research questions and their relevance. Inclusion and exclusion criteria are applied to narrow down the results, using data extraction forms designed to collect all the necessary information, synthesize data and summarize the results of each of the studies.

In the last phase, the documented results are analyzed and discussed in a structured way, leading to the conclusions of the SR. In addition, trends, limitations of the study and recommendations for future research are indicated.

It is important to highlight the inherent methodological limitations of the SR, in order to properly assess the importance of each of the phases indicated in Figure 1. One of the main alterations that affect this type of review is the one known as bias of publication. This is derived from the fact that many scientific works, mostly with negative results (those that do not find significant differences or with results contrary to the study hypothesis or what is usually established) are never published, take longer to do so or they are less cited in other publications. All this conditions the results of a bibliographic search and can lead to biased results.

\subsection{Research Questions}

The research questions should address the impact of AR technology on the training of spatial skills in the learning of STEM subjects. In this sense, there is a gap in the literature which this research aims to address in relation to AR research used for the purpose of improving students' spatial intelligence. Therefore, the following research questions (RQs) 
are posed regarding $A R$, spatial intelligence and its application in STEM educational settings (Table 1).

Table 1. RQs related to the application of AR in STEM education and their impact on students' spatial intelligence.

\begin{tabular}{cc}
\hline RQ1 & What impact does AR have on the development of spatial intelligence? \\
\hline RQ2 & What spatial skills have been assessed using AR? \\
\hline RQ3 & What was the effect of AR on STEM content learning? \\
\hline RQ4 & What kind of learning tools and AR resources have been used in the development \\
RQ5 & What spatial intelligence?
\end{tabular}

\subsection{Research Process}

In order to identify the primary studies that respond to the research questions, an exhaustive search for scientific articles was conducted in the three main bibliometric databases: Scopus, Web of Science and Google Scholar. According to the number, coverage and quality of citations of these databases, Scopus stands out for having a wide coverage of journals, compared to the high number of citations at the expense of the quality of Google Scholar, and the high quality at the expense of the decreasing number of citations of Web of Science [36,37].

The initial search for studies was done mainly on the basis of their title, abstract and keywords. We searched for the following keywords in English: "Augmented Reality" and "Augmenting Reality" in combination with "Spatial Intelligence", "Spatial Ability", "Spatial Abilities" and "Visuospatial Ability" linked to Education, Learning, Teaching, Instruction and Training. The search was conducted on 4 April 2021 and found 242 studies. Unpublished studies were not included because their assessment quality cannot be guaranteed in the absence of a peer review process.

\subsection{Inclusion and Exclusion Criteria}

Both authors reviewed the studies resulting from the initial search in order to identify those relevant to the paper. This allowed us to exclude research that did not address the purpose of the study or the previously stated requirements. To do so, we screened by selecting studies that met the following conditions: (a) empirical studies that measured the impact of AR on students' spatial intelligence as an outcome variable, (b) studies that provided sufficient descriptive statistical information, and (c) studies that included a pretest-posttest model in control and experimental groups.

\subsection{Data Collection}

For data collection, a data extraction document was designed to compile the information to address the research questions. Each article was read by the two researchers, who used the content analysis technique recommended by $\mathrm{Hsu}$ et al. [38] to extract the data. The data form allowed the following information to be collected from each of the primary studies: literature reference, educational level, content, spatial skills assessed, spatial intelligence tests used, hardware, software and educational resources used, benefits of AR on spatial intelligence, usefulness of AR in STEM learning and limitations of AR.

\subsection{Data Analysis}

In order to address the research questions, the evaluation criteria used in the data extraction forms for each selected study were linked to each of them. This tabulation allowed the studies to be structured according to their shared characteristics [11]. The list of evaluation criteria for the tabulation of data classified by research questions is shown in Table 2. 
Table 2. Evaluation criteria related to the research questions.

\begin{tabular}{cc}
\hline Research Question & Evaluation Criteria \\
\hline RQ1 & Benefits of AR on spatial intelligence \\
RQ2 & Spatial skills assessed \\
& Spatial intelligence tests used \\
RQ3 & Educational level \\
& Contents \\
RQ4 & Utility of AR in STEM learning \\
RQ5 & Hardware \\
& Software \\
& Educational Resources \\
\end{tabular}

\section{Results}

As already indicated in the methodology section, the articles selected for this SR were retrieved from the Scopus, Web of Science and Google Scholar databases. These databases were selected because they complied with the requirements of the protocol and with the filtering options where the specific parameters established were mechanized. As shown in Figure 2, the searches returned 242 articles from the databases, including 45 duplicates, which reduced the number of articles to 197. After reviewing their titles, keywords and abstracts, 103 articles were excluded because they did not meet the inclusion criteria in terms of the focus of the study. This left 94 articles to be assessed for eligibility, which were carefully examined for their relevance to the SR. Due to the exclusion criteria, 77 articles were excluded. Finally, a total of 17 scientific articles were analyzed or included in the review.

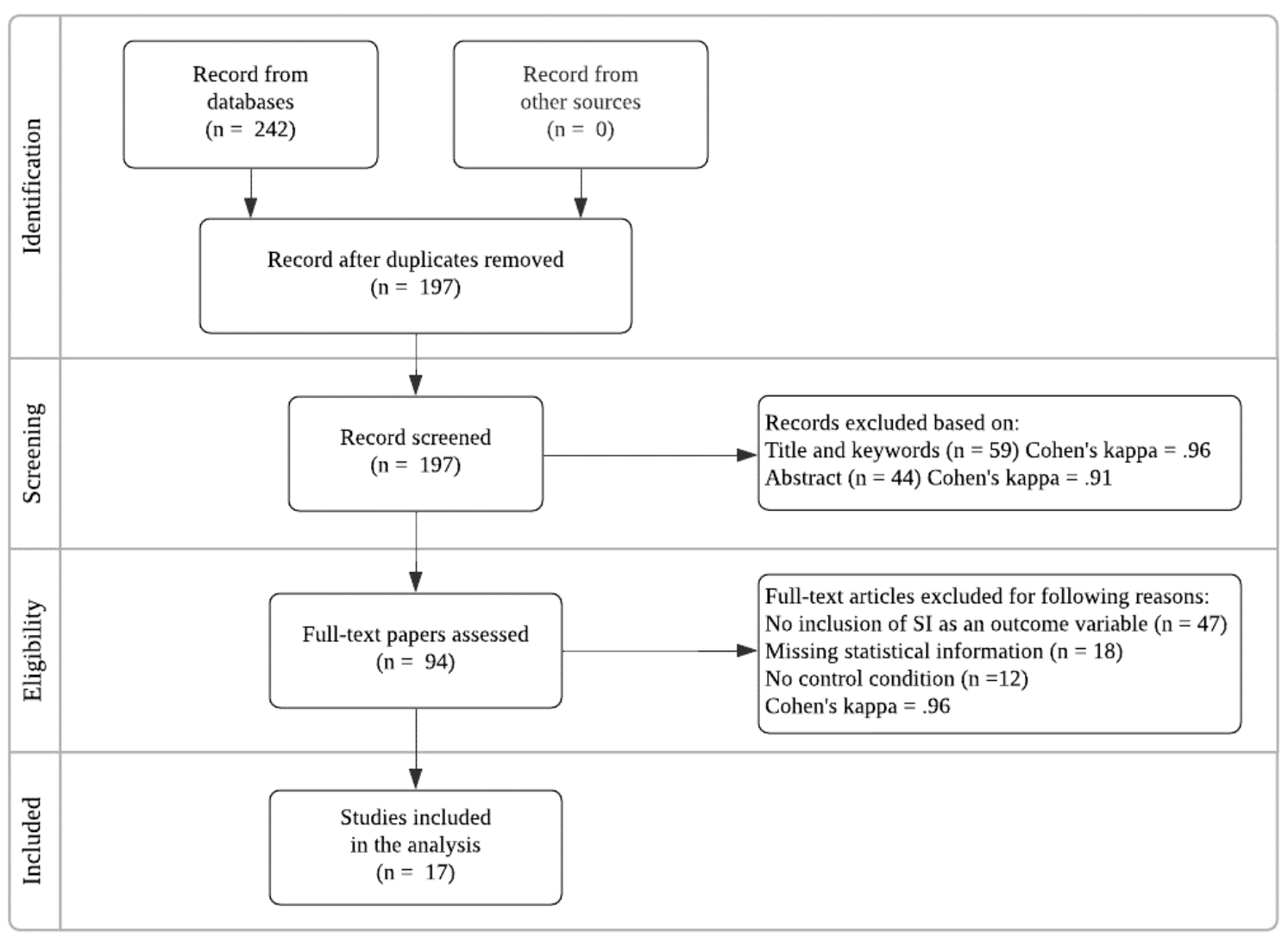

Figure 2. Flowchart of the study selection process. 
As it is usual in SRs of the literature, Cohen's kappa coefficient was used to verify the robustness between coders at each level of exclusion [39]. Values above 0.9 were found, which corresponds to almost perfect agreement between authors in the filtering and selection of studies; while any occasional disagreements were discussed and resolved by consensus.

The results obtained in a preliminary analysis show the number of research studies selected for this study related to AR and spatial intelligence in STEM subject areas according to their year of publication (Table 3). From 2002 to 2013, a very low and interrupted number of studies were published. It is observed that, from 2014 onwards, the research topic received greater and more permanent attention, although the number of papers is still very limited. The results suggest, in summary, that interest in this topic of study has increased from 2014 to 2021, and it is believed that this trend will continue in the forthcoming years. This finding is significant and will serve to guide future research in STEM knowledge areas.

Table 3. Number of articles published per year.

\begin{tabular}{cccc}
\hline Year & Articles & Year & Articles \\
\hline 2002 & 1 & 2016 & 1 \\
2006 & 1 & 2017 & 1 \\
2010 & 1 & 2018 & 1 \\
2011 & 1 & 2019 & 1 \\
2012 & 1 & 2020 & 3 \\
2014 & 1 & 2021 & 1 \\
2015 & 3 & & \\
\hline
\end{tabular}

Table 4 illustrates the distribution of the articles selected for the SR according to the country of the institutions linked to the lead researcher. It should be noted that the main countries that have contributed most in this area are Spain $[4,18,40-42]$ and Taiwan $[7,43,44]$. Of the total number of articles, eight were carried out thanks to funding from institutions or agencies, most notably the Spanish Ministry of Science and Innovation and the Austrian Science Fund.

Table 4. Number of articles published per country.

\begin{tabular}{cccc}
\hline Country & Articles & Country & Articles \\
\hline Spain & 5 & New Zealand & 1 \\
Taiwan & 3 & Indonesia & 1 \\
Austria & 2 & Peru & 1 \\
Germany & 1 & Malaysia & 1 \\
USA & 1 & Netherlands & 1 \\
\hline
\end{tabular}

The excerpt illustrated in Table 5 below corresponds to the analyzed articles that use AR to promote the development of spatial skills in students through different learning methodologies in different areas of STEM education and training. This table shows the most noteworthy experimental evidence of each of the scientific articles selected for the SR, according to the established evaluation criteria. 
Table 5. Evaluation Summary of key findings from selected studies.

\begin{tabular}{|c|c|c|c|c|c|c|c|}
\hline Reference & $\begin{array}{l}\text { Educational } \\
\text { Level. Study } \\
\text { Sample }\end{array}$ & $\begin{array}{l}\text { Spatial Skills } \\
\text { Assessed }\end{array}$ & $\begin{array}{c}\text { Spatial } \\
\text { Intelligence } \\
\text { Tests }\end{array}$ & $\begin{array}{l}\text { Hardware } \\
\text { Software }\end{array}$ & $\begin{array}{l}\text { Benefits of AR on } \\
\text { Spatial Intelligence }\end{array}$ & $\begin{array}{c}\text { Usefulness of } \\
\text { AR } \\
\text { for Learning }\end{array}$ & $\begin{array}{l}\text { Limitations } \\
\text { of AR }\end{array}$ \\
\hline $\begin{array}{l}\text { Bogomolova } \\
\text { et al., } 2020 \\
{[45]}\end{array}$ & $\begin{array}{l}\text { Higher } \\
\text { Education. } \\
\text { Medicine. } \\
\text { Anatomy. } \\
n=20\end{array}$ & $\begin{array}{c}\text { Spatial } \\
\text { Visualisation } \\
\text { and Mental } \\
\text { Rotation }\end{array}$ & $\begin{array}{c}\text { Mental Rotation } \\
\text { Test (MRT), } \\
\text { Paper Folding } \\
\text { Test (PFT), } \\
\text { Mechanical } \\
\text { Reasoning (MR) }\end{array}$ & $\begin{array}{l}\text { HoloLens }{ }^{\circledR} \text { Dy- } \\
\text { namicAnatomy }\end{array}$ & $\begin{array}{l}\text { Three-dimensional } \\
\text { anatomical models } \\
\text { that can be viewed } \\
\text { stereoscopically in } \\
\text { AR help to optimise } \\
\text { the acquisition of } \\
\text { anatomical learning } \\
\text { in students with low } \\
\text { visual spatial skills. }\end{array}$ & $\begin{array}{c}\text { Helps to } \\
\text { decrease } \\
\text { external } \\
\text { cognitive load } \\
\text { and facilitate a } \\
\text { better } \\
\text { understanding } \\
\text { of 3D anatomy } \\
\text { in students } \\
\text { with low } \\
\text { visuo-spatial } \\
\text { level. }\end{array}$ & $\begin{array}{l}\text { Unsettled } \\
\text { technology. In } \\
\text { order to detect } \\
\text { knowledge } \\
\text { retention, a } \\
\text { long-term test } \\
\text { would be } \\
\text { necessary. }\end{array}$ \\
\hline $\begin{array}{c}\text { Camba } \\
\text { et al., 2014 } \\
{[46]}\end{array}$ & $\begin{array}{c}\text { Higher } \\
\text { Education. } \\
\text { Design } \\
\text { Engineering. } \\
\text { Graphic } \\
\text { Expression. } \\
n=22\end{array}$ & $\begin{array}{c}\text { Spatial } \\
\text { Visualisation }\end{array}$ & $\begin{array}{l}\text { Psychometrically } \\
\text { validated } \\
\text { questionnaire }\end{array}$ & $\begin{array}{l}\text { Mobile devices. } \\
\text { AR-Engineering }\end{array}$ & $\begin{array}{l}\text { Graphic materials in } \\
\text { AR have a positive } \\
\text { impact on students' } \\
\text { visualisation and } \\
\text { spatial skills. }\end{array}$ & $\begin{array}{l}\text { AR technology } \\
\text { is especially } \\
\text { effective in } \\
\text { learning areas } \\
\text { with high } \\
\text { demand for } \\
\text { spatial skills, } \\
\text { such as graphic } \\
\text { expression. }\end{array}$ & $\begin{array}{c}\text { Graphics } \\
\text { processing } \\
\text { power is often } \\
\text { lower on } \\
\text { mobile devices. } \\
\text { Further } \\
\text { improvements } \\
\text { are needed in } \\
\text { integrating } \\
\text { more realistic } \\
\text { models, } \\
\text { animations and } \\
\text { markerless } \\
\text { activation. }\end{array}$ \\
\hline $\begin{array}{l}\text { Carbonell } \\
\text { \& Bermejo, } \\
2017[18]\end{array}$ & $\begin{array}{l}\text { Higher } \\
\text { Education. } \\
\text { Civil } \\
\text { Engineering. } \\
\text { Cartography. } \\
\quad n=63\end{array}$ & $\begin{array}{c}\text { Spatial } \\
\text { Visualisation } \\
\text { and Spatial } \\
\text { Interpretation }\end{array}$ & $\begin{array}{l}\text { Topographic } \\
\text { Map } \\
\text { Assessment } \\
\text { (TMA) }\end{array}$ & $\begin{array}{c}\text { Mobile devices: } \\
\text { tablets. } \\
\text { AR-media }^{\mathrm{TM}} \\
\text { ARrelief- }^{-} \\
\text {Workshop }\end{array}$ & $\begin{array}{l}\text { Participants } \\
\text { improved their } \\
\text { ability to interpret } \\
\text { highlights in a } \\
\text { statistically } \\
\text { significant way. }\end{array}$ & $\begin{array}{c}\text { AR is a } \\
\text { powerful tool } \\
\text { for viewing and } \\
\text { interpreting 3D } \\
\text { terrain, and } \\
\text { when } \\
\text { implemented } \\
\text { on a } \\
\text { touchscreen it } \\
\text { allows intuitive } \\
\text { user interaction. }\end{array}$ & $\begin{array}{l}\text { Shortage of } \\
\text { cartographic } \\
\text { material } \\
\text { incorporating } \\
\text { AR markers. }\end{array}$ \\
\hline $\begin{array}{l}\text { Chen et al., } \\
2011 \text { [7] }\end{array}$ & $\begin{array}{l}\text { Higher } \\
\text { Education. } \\
\text { Design } \\
\text { Engineering. } \\
n=35\end{array}$ & $\begin{array}{c}\text { Spatial } \\
\text { Visualisation } \\
\text { and Mental } \\
\text { Rotation }\end{array}$ & $\begin{array}{l}\text { Psychometrically } \\
\text { validated } \\
\text { questionnaire }\end{array}$ & $\begin{array}{l}\text { Computer with } \\
\text { webcam. } \\
\text { ARToolKitPlus }\end{array}$ & $\begin{array}{l}\text { The augmented } \\
\text { materials helped } \\
\text { participants with } \\
\text { poor spatial skills to } \\
\text { increase their spatial } \\
\text { understanding. }\end{array}$ & $\begin{array}{l}\text { Students can } \\
\text { observe } \\
\text { features of } \\
\text { models from } \\
\text { arbitrary views } \\
\text { to enhance their } \\
\text { learning. }\end{array}$ & $\begin{array}{l}\text { The effect of } \\
\text { using AR on } \\
\text { students with } \\
\text { high spatial } \\
\text { ability is } \\
\text { negligible. }\end{array}$ \\
\hline $\begin{array}{c}\text { Contero } \\
\text { et al., } 2012 \\
{[41]}\end{array}$ & $\begin{array}{c}\text { Higher } \\
\text { Education. } \\
\text { Design } \\
\text { Engineering. } \\
\text { Graphic } \\
\text { Expression. } \\
n=27\end{array}$ & $\begin{array}{c}\text { Spatial } \\
\text { Visualisation } \\
\text { and Mental } \\
\text { Rotation }\end{array}$ & $\begin{array}{c}\text { Differential } \\
\text { Aptitude Test: } \\
\text { Space Relations } \\
\text { (DAT: SR), } \\
\text { Mental Rotation } \\
\text { Test (MRT) }\end{array}$ & $\begin{array}{l}\text { Computer with } \\
\text { webcam. } \\
\text { AR-Books }\end{array}$ & $\begin{array}{l}\text { The experimental } \\
\text { group shows a } \\
\text { significant } \\
\text { improvement in } \\
\text { their levels of } \\
\text { spatial visualisation } \\
\text { and mental rotation } \\
\text { after using AR. The } \\
\text { interactivity of AR } \\
\text { applications } \\
\text { contributes to the } \\
\text { understanding of } \\
\text { the concept of space. }\end{array}$ & $\begin{array}{c}\text { Tangible } \\
\text { interaction with } \\
\text { augmented } \\
\text { content is an } \\
\text { added value } \\
\text { that is reflected } \\
\text { in the learning } \\
\text { outcomes. }\end{array}$ & $\begin{array}{l}\text { Problems may } \\
\text { arise when } \\
\text { students } \\
\text { request more } \\
\text { AR material to } \\
\text { support them in } \\
\text { subsequent } \\
\text { courses. A great } \\
\text { deal of effort is } \\
\text { required to } \\
\text { create } \\
\text { augmented } \\
\text { learning } \\
\text { content. }\end{array}$ \\
\hline $\begin{array}{l}\text { Del Cerro } \\
\& \text { Morales, } \\
2021 \text { [4] }\end{array}$ & $\begin{array}{l}\text { Secondary } \\
\text { Education. } \\
\text { Mathematics. } \\
\text { Calculus. } \\
n=23\end{array}$ & $\begin{array}{c}\text { Spatial } \\
\text { Visualisation } \\
\text { and Mental } \\
\text { Rotation }\end{array}$ & $\begin{array}{l}\text { Purdue Spatial } \\
\text { Visualization } \\
\text { Test: Rotations } \\
\text { (PSVT: R) }\end{array}$ & $\begin{array}{l}\text { Mobile devices. } \\
\text { Geogebra AR }\end{array}$ & $\begin{array}{c}\text { Significant } \\
\text { improvement in the } \\
\text { levels of academic } \\
\text { achievement and } \\
\text { spatial intelligence } \\
\text { of students exposed } \\
\text { to it. }\end{array}$ & $\begin{array}{l}\text { It promotes } \\
\text { proactive and } \\
\text { delocalised } \\
\text { learning, } \\
\text { verifying and } \\
\text { accurately } \\
\text { evaluating the } \\
\text { results } \\
\text { autonomously. } \\
\text { The factors of } \\
\text { attention and } \\
\text { motivation } \\
\text { have a positive } \\
\text { impact on } \\
\text { learning. }\end{array}$ & $\begin{array}{c}\text { In order to } \\
\text { detect } \\
\text { knowledge } \\
\text { retention, a } \\
\text { long-term test } \\
\text { would be } \\
\text { necessary. } \\
\text { Better teacher } \\
\text { training is } \\
\text { needed to } \\
\text { design teaching } \\
\text { materials in AR. }\end{array}$ \\
\hline
\end{tabular}


Table 5. Cont.

\begin{tabular}{|c|c|c|c|c|c|c|c|}
\hline Reference & $\begin{array}{l}\text { Educational } \\
\text { Level. Study } \\
\text { Sample }\end{array}$ & $\begin{array}{l}\text { Spatial Skills } \\
\text { Assessed }\end{array}$ & $\begin{array}{c}\text { Spatial } \\
\text { Intelligence } \\
\text { Tests }\end{array}$ & $\begin{array}{l}\text { Hardware } \\
\text { Software }\end{array}$ & $\begin{array}{l}\text { Benefits of AR on } \\
\text { Spatial Intelligence }\end{array}$ & $\begin{array}{c}\text { Usefulness of } \\
\text { AR } \\
\text { for Learning }\end{array}$ & $\begin{array}{l}\text { Limitations } \\
\text { of AR }\end{array}$ \\
\hline $\begin{array}{l}\text { Dünser } \\
\text { et al., 2006 } \\
\text { [23] }\end{array}$ & $\begin{array}{l}\text { Secondary } \\
\text { Education. } \\
\text { Mathematics. } \\
\text { Geometry. } \\
\quad n=47\end{array}$ & $\begin{array}{c}\text { Spatial } \\
\text { Visualisation, } \\
\text { Mental Rotation } \\
\text { and Spatial } \\
\text { Orientation }\end{array}$ & $\begin{array}{c}\text { Differential } \\
\text { Aptitude Test: } \\
\text { Space Relations } \\
\text { (DAT: SR), } \\
\text { MentalCutting } \\
\text { Test (MCT), } \\
\text { Mental Rotation } \\
\text { Test (MRT), } \\
\text { Objective } \\
\text { Perspective Test } \\
\text { (OPT) }\end{array}$ & $\begin{array}{c}\text { HeadMounted } \\
\text { Display (HMD), } \\
\text { Computer. } \\
\text { Construct3D, } \\
\text { CAD3D }\end{array}$ & $\begin{array}{l}\text { AR is a very useful } \\
\text { tool for training } \\
\text { spatial skills. } \\
\text { Applications can be } \\
\text { developed to } \\
\text { examine different } \\
\text { spatial aspects and } \\
\text { behaviours. }\end{array}$ & $\begin{array}{c}\text { The } \\
\text { interactivity of } \\
\text { AR provides a } \\
\text { learning } \\
\text { stimulus that } \\
\text { enhances } \\
\text { students' } \\
\text { assimilation of } \\
\text { content. } \\
\text { External } \\
\text { cognitive load } \\
\text { is reduced, } \\
\text { users do not } \\
\text { have to } \\
\text { mentally } \\
\text { interpret or } \\
\text { transform the } \\
\text { content. }\end{array}$ & $\begin{array}{l}\text { The measures } \\
\text { of spatial ability } \\
\text { taken do not } \\
\text { cover all the } \\
\text { capabilities that } \\
\text { are used when } \\
\text { working in } \\
\text { three- } \\
\text { dimensional } \\
\text { space. } \\
\text { Therefore, new } \\
\text { tools to } \\
\text { measure spatial } \\
\text { intelligence } \\
\text { directly in 3D } \\
\text { would be } \\
\text { desirable. }\end{array}$ \\
\hline $\begin{array}{l}\text { Gómez- } \\
\text { Tone et al., } \\
2020 \text { [47] }\end{array}$ & $\begin{array}{c}\text { Higher } \\
\text { Education. } \\
\text { Design } \\
\text { Engineering. } \\
\text { Graphic } \\
\text { Expression. } \\
n=31\end{array}$ & $\begin{array}{c}\text { Spatial } \\
\text { Visualisation } \\
\text { and Mental } \\
\text { Rotation }\end{array}$ & $\begin{array}{c}\text { Differential } \\
\text { Aptitude Test: } \\
\text { Space Relations } \\
\text { (DAT: SR), } \\
\text { Mental Rotation } \\
\text { Test (MRT) }\end{array}$ & $\begin{array}{l}\text { Computer with } \\
\text { webcam. } \\
\text { AR-Books }\end{array}$ & $\begin{array}{l}\text { The experimental } \\
\text { groups achieved } \\
\text { significant gains in } \\
\text { their spatial ability } \\
\text { level, despite being } \\
\text { students from } \\
\text { different countries } \\
\text { whose initial level } \\
\text { of spatial skills were } \\
\text { different. No } \\
\text { differences were } \\
\text { detected in any of } \\
\text { the experimental } \\
\text { groups for the } \\
\text { gender factor. }\end{array}$ & $\begin{array}{c}\text { Poorly } \\
\text { developed } \\
\text { spatial skills act } \\
\text { as a barrier to } \\
\text { learning } \\
\text { achievement in } \\
\text { STEM subjects. } \\
\text { AR has a direct } \\
\text { relationship } \\
\text { between } \\
\text { academic } \\
\text { performance, } \\
\text { motivation and } \\
\text { self-learning. }\end{array}$ & $\begin{array}{l}\text { The experience } \\
\text { could have } \\
\text { been carried } \\
\text { out over several } \\
\text { semesters in } \\
\text { order to analyse } \\
\text { how it might } \\
\text { affect student } \\
\text { retention } \\
\text { during the } \\
\text { course. Lack of } \\
\text { available } \\
\text { resources and } \\
\text { teacher training } \\
\text { opportunities. }\end{array}$ \\
\hline $\begin{array}{l}\text { Gutiérrez } \\
\text { et al., 2016 } \\
\text { [42] }\end{array}$ & $\begin{array}{c}\text { Higher } \\
\text { Education. } \\
\text { Mechanical } \\
\text { Engineering. } \\
\text { Graphic } \\
\text { Expression. } \\
n=50\end{array}$ & $\begin{array}{c}\text { Spatial } \\
\text { Visualisation } \\
\text { and Mental } \\
\text { Rotation }\end{array}$ & $\begin{array}{c}\text { Differential } \\
\text { Aptitude Test: } \\
\text { Space Relations } \\
\text { (DAT: SR), } \\
\text { Purdue Spatial } \\
\text { Visualization } \\
\text { Test: Rotations } \\
\text { (PSVT: R) }\end{array}$ & $\begin{array}{l}\text { Mobile devices. } \\
\text { DiedricAR }\end{array}$ & $\begin{array}{l}\text { Positive impact on } \\
\text { students' spatial } \\
\text { skills achieved. }\end{array}$ & $\begin{array}{l}\text { Relevant results } \\
\text { were obtained } \\
\text { in the learning } \\
\text { of descriptive } \\
\text { geometry, in } \\
\text { addition to } \\
\text { ubiquity. } \\
\text { Flexible access } \\
\text { to learning } \\
\text { outside the } \\
\text { classroom. }\end{array}$ & $\begin{array}{l}\text { It would be } \\
\text { interesting to } \\
\text { include } \\
\text { self-assessment } \\
\text { tests in the app. }\end{array}$ \\
\hline $\begin{array}{c}\text { Habig, } 2020 \\
{[48]}\end{array}$ & $\begin{array}{c}\text { Higher } \\
\text { Education. } \\
\text { Chemistry. } \\
\text { Organic } \\
\text { Chemistry. } \\
n=31\end{array}$ & $\begin{array}{c}\text { Spatial } \\
\text { Visualisation } \\
\text { and Mental } \\
\text { Rotation }\end{array}$ & $\begin{array}{l}\text { Purdue Spatial } \\
\text { Visualization } \\
\text { Test: Rotations } \\
\text { (PSVT: R) }\end{array}$ & $\begin{array}{l}\text { Mobile devices. } \\
\text { AR-Chemistry } \\
\text { (ARC) }\end{array}$ & $\begin{array}{l}\text { AR technology is } \\
\text { useful for } \\
\text { enhancing learning } \\
\text { processes, reducing } \\
\text { the cognitive load of } \\
\text { students with low } \\
\text { spatial skills. AR } \\
\text { provides a } \\
\text { promising approach } \\
\text { for visualisation and } \\
\text { learning of complex } \\
\text { concepts. }\end{array}$ & $\begin{array}{l}\text { Students who } \\
\text { used AR scored } \\
\text { higher on } \\
\text { stereochemistry } \\
\text { content than } \\
\text { those who } \\
\text { learned from } \\
\text { textbooks and } \\
\text { notes. }\end{array}$ & $\begin{array}{c}\text { AR } \\
\text { representations } \\
\text { in instructional } \\
\text { material should } \\
\text { be better } \\
\text { implemented, } \\
\text { establishing } \\
\text { principles of } \\
\text { design and } \\
\text { integration of } \\
\text { activities. }\end{array}$ \\
\hline $\begin{array}{l}\text { Kaufmann } \\
\text { \& Schmal- } \\
\text { stieg, } 2002 \\
\text { [1] }\end{array}$ & $\begin{array}{l}\text { Secondary } \\
\text { Education. } \\
\text { Mathematics. } \\
\text { Geometry. } \\
n=14\end{array}$ & $\begin{array}{c}\text { Spatial } \\
\text { Visualisation, } \\
\text { Mental Rotation } \\
\text { and Spatial } \\
\text { Orientation. }\end{array}$ & $\begin{array}{l}\text { Psychometrically } \\
\text { validated } \\
\text { questionnaire }\end{array}$ & $\begin{array}{l}\text { Head Mounted } \\
\text { Display (HMD), } \\
\text { Computer. } \\
\text { Construct3D, } \\
\text { CAD3D }\end{array}$ & $\begin{array}{l}\text { It improves } \\
\text { students' spatial } \\
\text { skills. Complex } \\
\text { three-dimensional } \\
\text { spatial relationships } \\
\text { are directly visible. }\end{array}$ & $\begin{array}{c}\text { Improves } \\
\text { understanding } \\
\text { of three- } \\
\text { dimensional } \\
\text { geometry. } \\
\text { Encourages ex- } \\
\text { perimentation } \\
\text { with geometric } \\
\text { constructions. } \\
\text { Learning by } \\
\text { doing. }\end{array}$ & $\begin{array}{c}\text { Creating } \\
\text { educational } \\
\text { content for an } \\
\text { interactive } \\
\text { system is at } \\
\text { least as difficult } \\
\text { as creating } \\
\text { textbooks and } \\
\text { will require a } \\
\text { considerable } \\
\text { amount of time } \\
\text { and work. }\end{array}$ \\
\hline
\end{tabular}


Table 5. Cont.

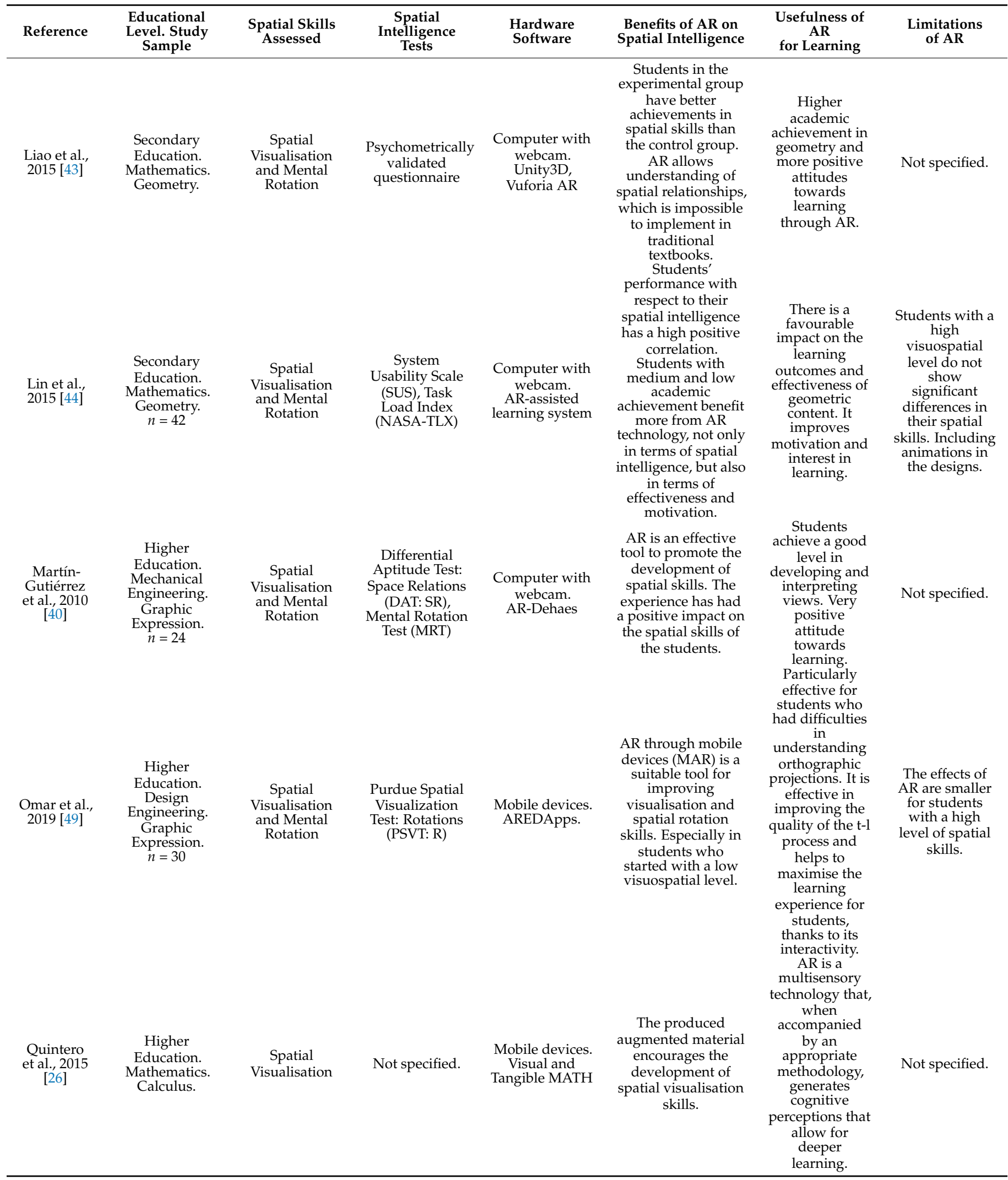


Table 5. Cont.

\begin{tabular}{|c|c|c|c|c|c|c|c|}
\hline Reference & $\begin{array}{l}\text { Educational } \\
\text { Level. Study } \\
\text { Sample }\end{array}$ & $\begin{array}{l}\text { Spatial Skills } \\
\text { Assessed }\end{array}$ & $\begin{array}{c}\text { Spatial } \\
\text { Intelligence } \\
\text { Tests }\end{array}$ & $\begin{array}{l}\text { Hardware } \\
\text { Software }\end{array}$ & $\begin{array}{l}\text { Benefits of AR on } \\
\text { Spatial Intelligence }\end{array}$ & $\begin{array}{c}\text { Usefulness of } \\
\text { AR } \\
\text { for Learning }\end{array}$ & $\begin{array}{l}\text { Limitations } \\
\text { of AR }\end{array}$ \\
\hline $\begin{array}{l}\text { Rohendi } \\
\text { et al., 2018 } \\
\text { [50] }\end{array}$ & $\begin{array}{l}\text { Secondary } \\
\text { Education. } \\
\text { Mathematics. } \\
\text { Geometry. }\end{array}$ & $\begin{array}{c}\text { Spatial } \\
\text { Visualisation }\end{array}$ & $\begin{array}{l}\text { Psychometrically } \\
\text { validated } \\
\text { questionnaire }\end{array}$ & $\begin{array}{c}\text { Mobile devices. } \\
\text { Vuforia }\end{array}$ & $\begin{array}{l}\text { Improvements in } \\
\text { spatial intelligence } \\
\text { are accentuated in } \\
\text { students with } \\
\text { medium-low spatial } \\
\text { skills. }\end{array}$ & $\begin{array}{l}\text { AR makes it } \\
\text { easier for } \\
\text { students to } \\
\text { understand } \\
\text { three- } \\
\text { dimensional } \\
\text { geometric } \\
\text { objects by being } \\
\text { able to rotate } \\
\text { three- } \\
\text { dimensional } \\
\text { objects freely, } \\
\text { making them } \\
\text { easier to view } \\
\text { from various } \\
\text { perspectives. }\end{array}$ & $\begin{array}{l}\text { To date, the use } \\
\text { of learning } \\
\text { through AR } \\
\text { technology is } \\
\text { still very } \\
\text { limited. }\end{array}$ \\
\hline
\end{tabular}

\section{Discussion}

For the purposes of the selection process described above and the evaluation criteria established for the conduct of the SR, the data collected and discussed in descriptive form are presented in different tables with respect to the research questions listed in Table 1.

\subsection{Impact of $A R$ on the Development of Spatial Intelligence (RQ1)}

The first evaluation criteria analyzed in the SR of the literature are related to the benefits of AR technology in improving the spatial skills of students exposed to it. After extracting the data from each of the chosen articles, they are linked to the identifying benefits in the form of absolute frequency in Table 6.

Table 6. Benefits of AR in the development of spatial intelligence.

\begin{tabular}{ccc}
\hline Key Benefits & $\mathbf{n}_{\mathbf{i}}$ & Sample Research \\
\hline $\begin{array}{c}\text { Improves spatial intelligence } \\
\text { It allows you to look at models from } \\
\text { different perspectives }\end{array}$ & 17 & Camba et al., 2014 [46] \\
$\begin{array}{c}\text { Helps to visualize abstract concepts } \\
\text { Greater benefit for users with low } \\
\text { visuospatial abilities }\end{array}$ & 70 & Martín-Gutiérrez et al., 2010 [40] \\
$\begin{array}{c}\text { Contributes to the understanding of the } \\
\text { concept of space }\end{array}$ & 7 & Bogomolova et al., 2020 [45] \\
Reduces cognitive load & 3 & Rohendi et al., 2018 [50] \\
\hline
\end{tabular}

The results of the review indicate unanimously (17 out of 17 ) that the use of AR tools in the classroom correlated with an improvement in students' spatial intelligence. This finding is supported by the quantitative results of the reviewed articles, where the experimental groups achieved significant gains in their spatial skills. Therefore, according to these results, we can affirm that AR technology is a tool that, used with an appropriate methodology and in a suitable context, has a positive impact on the spatial intelligence of students in STEM subjects.

Likewise, the potential effects of AR as a tool for visualizing abstract concepts alluded to in different articles (7 out of 17) is related to the concept of tangibility, since AR generally allows interactions with objects. The teaching-learning processes can be explained more easily when we use physical objects, because these objects are not only a representation for students, but also encourage proactive learning through their tangibility. Both the use of physical objects and their semantic representations, as well as their spatial arrangement, help students to understand and learn abstract content with a high visual load through manipulation. In addition, AR provides the ability to help students develop a deeper 
understanding of the concept of space [41], allowing the user to interact with it through immersive learning.

Similarly, some researchers reported that specific learning outcomes were related to the reduced cognitive weight of content with a high visual load (3 of 17). For example, Habig [48] compared effort and mental workload scores through ANOVA model analyses, which resulted in a significant difference in terms of effort and mental workload between the experimental and control groups. We strongly believe that this finding is related to the tangibility that AR provides by allowing students to interact with different materials, being able to observe them from different angles, as indicated by the vast majority of reviewed articles (10 out of 17). This functionality of $A R$ as a tool to support spatial visualization could be associated with the studies that reveal that the impact is especially significant in students who started with a low level of visuospatial skills (7 out of 17) when this technology was integrated.

\subsection{Spatial Skills Assessed through the Use of AR (RQ2)}

Thus far, there is no standardized test to measure spatial intelligence as a unit, so the assessment of spatial intelligence is made on the basis of the different skills that comprise it. For this reason, different specific standardized tests are used to provide relevant information about the different subcomponents of spatial intelligence. It is important for researchers to know what aspect they want to assess, so that they select the relevant tests with solid evidence of reliability and validity according to the objectives of their work.

After extracting the data from the studies corresponding to the assessments conducted by the researchers, they were organized into two categories, distinguishing between the spatial skills assessed and the validated assessment tests used. The results are presented descriptively in Table 7.

Table 7. Assessment of spatial skills.

\begin{tabular}{|c|c|c|c|}
\hline Category & Subcategory & $\mathbf{n}_{\mathbf{i}}$ & Sample Research \\
\hline \multirow{4}{*}{ Spatial skills } & Spatial visualization & 17 & Gutiérrez et al., 2016 [42] \\
\hline & Mental Rotation & 13 & Gómez-Tone et al., 2020 [47] \\
\hline & Spatial Orientation & 2 & Dünser et al., 2006 [23] \\
\hline & Spatial Interpretation & 1 & Carbonell and Bermejo, 2017 [18] \\
\hline \multirow{11}{*}{ Tests } & Mental Rotation Test (MRT) & 5 & Martín-Gutiérrez et al., 2010 [40] \\
\hline & $\begin{array}{c}\text { Differential Aptitude Test: Space } \\
\text { Relations (DAT: SR) }\end{array}$ & 5 & Contero et al., 2012 [41] \\
\hline & $\begin{array}{c}\text { Psychometrically validated } \\
\text { questionnaire }\end{array}$ & 5 & Chen et al., 2011 [7] \\
\hline & $\begin{array}{l}\text { Purdue Spatial Visualization } \\
\text { Test: Rotations (PSVT: R) }\end{array}$ & 4 & Del Cerro and Morales, 2021 [4] \\
\hline & Mental Cutting Test (MCT) & 1 & Dünser et al., 2006 [23] \\
\hline & Objective Perspective Test (OPT) & 1 & Dünser et al., 2006 [23] \\
\hline & System Usability Scale (SUS) & 1 & Lin et al., 2015 [44] \\
\hline & Task Load Index (NASA-TLX) & 1 & Lin et al., 2015 [44] \\
\hline & $\begin{array}{c}\text { Topographic Map } \\
\text { Assessment (TMA) }\end{array}$ & 1 & Carbonell and Bermejo, 2017 [18] \\
\hline & Paper Folding Test (PFT) & 1 & Bogomolova et al., 2020 [45] \\
\hline & Mechanical Reasoning (MR) & 1 & Bogomolova et al., 2020 [45] \\
\hline
\end{tabular}

Generally, studies on spatial intelligence tend to follow two lines of research regarding the definition of spatial skills. The first is composed of three skills: spatial perception, spatial orientation or mental rotation and spatial visualization. The second is composed of two skills: spatial visualization and mental rotation or spatial relation [51]. 
In relation to the above, after using methodological proposals integrating AR technology in the classroom or outside the classroom, the authors measure the level of achievement in different spatial skills, with the assessment of spatial visualization (17 out of 17) and mental rotation (12 out of 17) predominating in the studies selected for SR. Only two studies [18,23] assess the skill of spatial orientation; therefore, most authors apply the line of research that focuses on analyzing the skills of spatial visualization and mental rotation to the study of spatial intelligence.

Spatial visualization [52] denotes the ability to perceive and mentally recreate twoand three-dimensional objects or models. Several authors $[53,54]$ use the term spatial visualization to indicate the processes and abilities of individuals to perform tasks that require seeing or mentally imagining spatial geometric objects, as well as relating these objects and performing geometric operations or transformations with them.

Shepard and Metzler [55] define mental rotation ability as the cognitive ability to rotate ideal representations of dimensional and/or three-dimensional objects or models and this can be described as the movement of representations by the brain to help conceive each of their views or perspectives with respect to a rotation.

On the other hand, there are several standardized tests to measure a person's ability in the first two stages of spatial development. Firstly, the most commonly used in the selected items are the Mental Rotation Test (MRT) and the Differential Aptitude Test: Space Relations (DAT: SR). Both assessment tools measure students' cognitive ability to perform different processes of converting two-dimensional information into three-dimensional information in the absence of stimuli.

Specifically, the MRT test, which was developed by Vandenberg and Kuse [56], is one of the tests with the highest level of acceptance and applicability worldwide [57]. The MRT test is designed to measure the ability of mental rotation, through a test consisting of 20 items, and in each of them, the user must choose the correct figure from four options (A-D) that represents the rotation of the pattern figure (Figure 3).

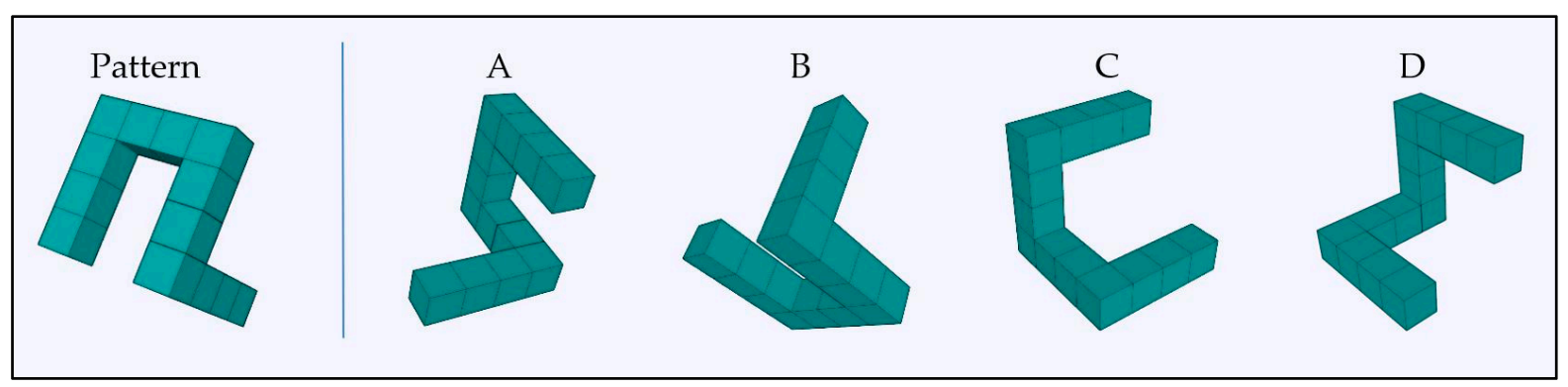

Figure 3. MRT sample question.

In a similar way, the DAT: SR questionnaire by Bennet et al. [58], specifically measures the student's ability to move from the 2D to the 3D domain [59]. It consists of 50 items that require the learner to mentally fold the $2 \mathrm{D}$ pattern and choose from four alternatives (A-D), the correct 3D object which would result, given the original 2D pattern (Figure 4).

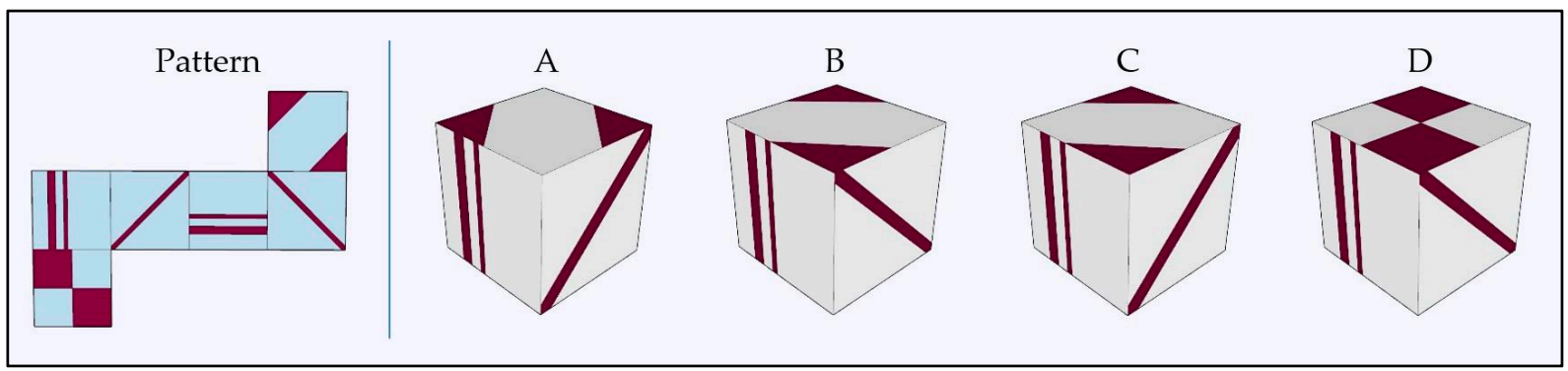

Figure 4. DAT: SR sample question. 
Secondly, in terms of the most commonly used spatial assessment tools, we find the PSVT: R test, used to measure the level of visualization and spatial rotation skills [60]. This test consists of 12 items, in which the student is asked to indicate for each, which of the options (A-E) is the correct view representing the next rotation in the pattern (Figure 5). In most articles, it has been used as an assessment tool at the beginning and at the end of the experience in experimental and control groups, following a pretest and posttest design, in order to evaluate the impact on students' spatial intelligence through classroom experience using AR in STEM subjects. In a study by Sorby [61], the PSVT: R was shown to be a tool that helps to significantly measure the spatial skills of students studying engineering.

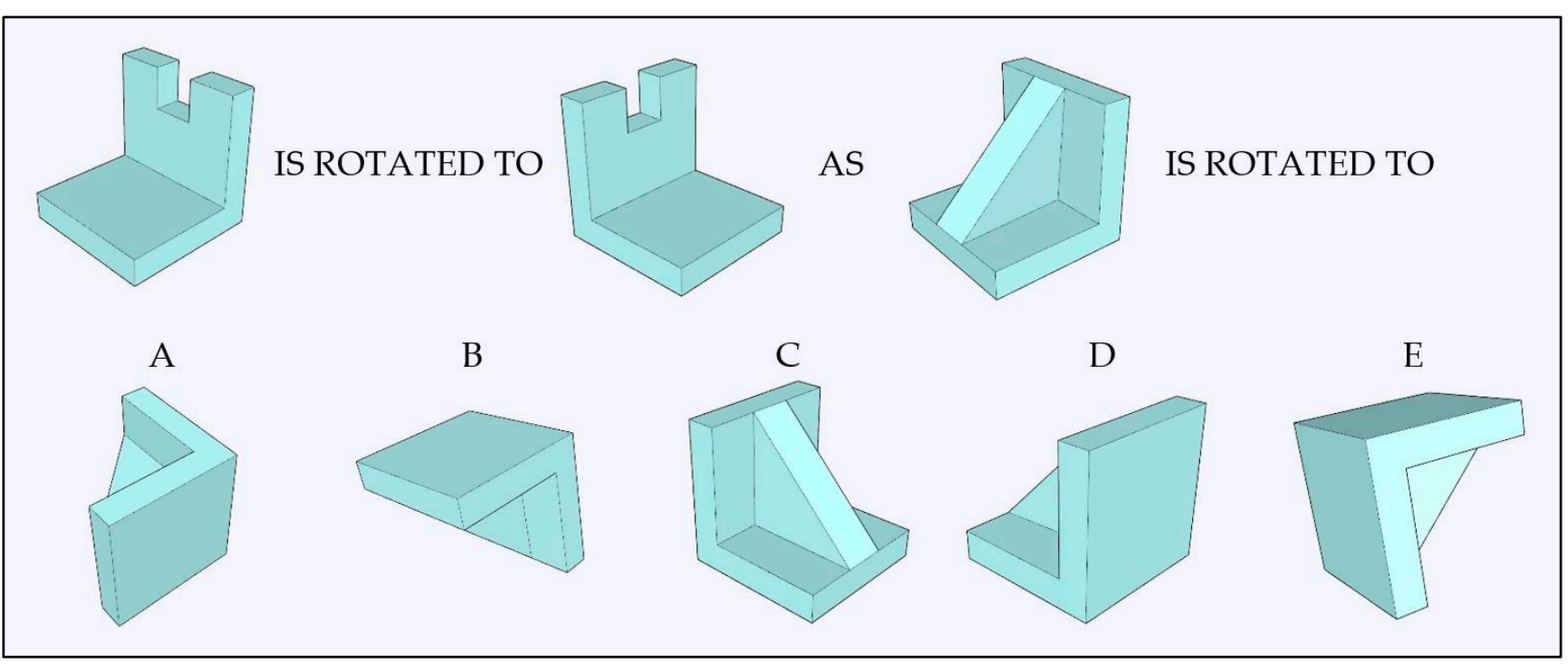

Figure 5. PSVT: R sample question.

Finally, other specific tests with minimal frequency in the STEM subject area are used, related to very specific content, disciplines and methodologies. In the research focused on the study of geometry developed by Dünser et al. [23], the Mental Cutting Test (MCT) and Objective Perspective Test (OPT) are used as assessment tools. In other work in the same field, Lin et al. [44] use the System Usability Scale (SUS) and NASA Task Load Index (NASATLX) assessment tools to validate their questionnaires. In order to assess the effectiveness of Civil Engineering students in the study of cartography, Carbonell and Bermejo [18] use the specific questionnaire Topographic Map Assessment (TMA) for the spatial interpretation of topographic reliefs. On the other hand, researchers Bogomolova et al. [45] apply the Paper Folding Test (PFT) and Mechanical Reasoning (MR) to assess the stereoscopic vision skills of medical students in the acquisition of anatomical learning.

\subsection{Contributions of AR in STEM Content Learning (RQ3)}

From a pedagogical point of view, the most outstanding contributions of AR in STEM subjects are: its contribution to understanding content with a high visual load, the improvement in learning outcomes, the promotion of proactive learning, the greater effectiveness in students with a low visuospatial level and the enhancement of motivation and interest in learning (Table 8). 
Table 8. Pedagogical benefits of AR in STEM content learning.

\begin{tabular}{ccc}
\hline Key Contributions & $\mathbf{n}_{\mathbf{i}}$ & Sample Research \\
\hline Helps to understand content with a high visual load & 11 & Camba et al., 2014 [46] \\
Improves learning outcomes & 8 & Habig, 2020 [48] \\
Promotes proactive learning & 8 & Gómez-Tone et al., 2020 [47] \\
More effective in students with low visuospatial skills & 7 & Omar et al., 2019 [49] \\
Boosts motivation and interest in learning & 6 & Liao et al., 2015 [43] \\
\hline
\end{tabular}

Most of the studies (11 out of 17) agreed that AR technology helps students to understand content with a high visual load in STEM subjects. One example is the research conducted in the area of graphic expression by Martín-Gutiérrez et al. [40], who presented an augmented book training system called AR-Dehaes, designed to provide virtual models that can help engineering students perform visualization and spatial rotation tasks in order to promote the development of their spatial intelligence. The authors conclude that the validated study had a positive impact on learning with respect to the methodology that makes use of traditional textbooks. Similar research by Dünser et al. [23] and Bogomolova et al. [45], indicates that AR provides learning stimuli that enhance students' assimilation of content. This implies a reduction of external cognitive load, and increases students' ability to focus their attention on the most relevant information, using it as a support tool for cognitive transformations and interpretations. They also add that AR technology is especially effective in learning content with a high demand for spatial skills, such as graphic expression [46].

In addition, numerous articles (8 out of 17) highlight the improvement of students' academic performance and learning outcomes after integrating augmented materials into STEM educational environments. According to work in the areas of descriptive geometry [42-44] and stereochemistry [48], students who used AR technology obtained higher grades than those who used a textbook and notebook learning methodology. This is because AR allows students to visualize abstract concepts virtually, which cannot be easily seen in a real environment.

Another pedagogical benefit of AR is the possibility it offers to users with a low level of visuospatial skills (7 out of 17). Through it, they can interact and visualize models from different perspectives, which is closely related to an improvement in students' learning satisfaction, helping students with difficulties to solve specific problems and memorize learning materials better. One example is the work by Omar et al. [49], where they show that AR is especially effective for students who started with low spatial skills and had difficulties in understanding orthographic projections. Experiences by Bogomolova et al. [45] and Habig [48] in learning anatomy and organic chemistry respectively, conclude that AR technology is useful for improving learning processes, reducing the external cognitive load of students with low spatial skills, and it highlights the relevance that AR can have for visualizing and understanding complex concepts.

An additional advantage in learning is that AR technology allows us to virtually recreate any physical object and, through a tangible interface, we are able to manipulate it so as to obtain information from it with the aim of promoting proactive learning ( 8 of 17). This is because AR makes it possible to replace physical resources with virtual objects, which leads us to understand that we are facing an educational tool with great potential to improve the assimilation of content and teaching-learning processes in the coming years in any area; but, fundamentally, in all content where spatial intelligence is especially relevant, as it is in the case in STEM subjects [29]. Likewise, AR technology allows the user to interact and observe the characteristics of three-dimensional models from different arbitrary views to increase their spatial understanding [7].

Gómez-Tone et al. [47], points out that tangible interactions with objects in AR is an added learning value that contributes to improve the understanding of the concept of space. Very similar results have been obtained in the research developed by Bogomolova et al. [45] 
on an application of three-dimensional stereoscopic anatomical models, highlighting the advantages of AR to enhance collaborative and proactive learning.

In addition to enhancing students' proactive learning, AR awakens their inquisitiveness and need to know more. Students have the possibility of verifying and evaluating the correctness and accuracy of the results of their exercises autonomously through AR. In the experiment for assay carried out by del Cerro and Morales [4] for the study of mathematical functions, the experimental group could draw, analyze and interpret several graphs at the same time through AR without having to perform algebraic calculations, create a table of values or trace each of them by hand. This meant that they generally completed the activities proposed in class in less time than the control group, a factor that may have contributed to the deepening of content and a higher score in the specific content test than that obtained by the control group.

For all these reasons, it is essential to encourage tangible and proactive learning. These pedagogical methodologies based on learning-by-doing, advocate experimental experiences focused on the acquisition of knowledge that generate more meaningful and deeper learning.

The results of the review also indicate that AR can improve students' motivation, interest and positive attitude towards learning (6 out of 17). These factors have a direct relationship in improving students' academic performance, and in learning both basic and abstract concepts $[4,40,43,44,47]$.

Regarding the educational level, of the 17 research projects selected for SR, 11 were carried out at higher education levels and 6 at secondary education levels, with a predominance of methodological applications of AR in the areas of technology, graphic expression (technical drawing) and mathematics, which demonstrates a generalized trend in the field of spatial skills training in higher education students.

\subsection{AR Tools and Resources for the Development of Spatial Intelligence (RQ4)}

As recorded in Table 5, the AR devices or systems used by the selected studies are: mobile devices (8 out of 17), computer with webcam (6 out of 17) and Head Mounted Display (HMD) glasses or headsets (3 out of 17 ).

HMDs were used in the first experiences with AR in education in the 2000s $[1,23]$ as display devices that allowed virtual objects to be superimposed on a screen located very close to the eyes. However, due to their high cost, the lack of widespread technology required for their use and the limitations of software development kits (SDKs), HMDs did not become widespread in society. After years of limited interest from developers and society, technology giants are now bringing HMDs back into education, enabling the development of learning experiences through real-time 3D models for students to learn in an immersive way. An example of this is the experience with the HoloLens device [45], which allows medical holograms to be viewed stereoscopically in real time with the human body. Everything points to the fact that in a few years this type of device will be integrated into people's daily lives and its use as a learning tool will become more widespread.

However, HMDs are currently an exception within AR in education, as the vast majority of applications are developed for mobile devices due to their omnipresence in society. It can be observed that from 2011 onwards, mobile devices began to be integrated as a hardware platform for carrying out AR experiences, dethroning the experiences that up to that date used computers with webcams. The work of Camba et al. [46] indicates that the graphics processing power is usually lower on mobile devices compared to personal computers, which can limit the integration of more realistic, detailed models, with animations and the activation of AR without marks (markerless). On the other hand, the work of Gutiérrez et al. [42] highlights some benefits of these devices for mobile learning (m-learning) or delocalized learning, such as ubiquity and flexible access outside the classroom. The learning methodology through m-learning offers us an accessible, interactive, collaborative and simple way to acquire knowledge never seen before, breaking the space-time barrier. However, it also has some minor disadvantages, since it can lead 
to a certain difficulty in reading texts, it depends on a network connection, and, the less controllable risk, the level of distraction of students in front of mobile devices can be very high. For all these reasons, and after conducting the SR, it is worth highlighting the role of mobile AR learning (MAR), which has the ability to help break down economic barriers, economic constraints and rural-urban differences. In addition, the continuous improvement of mobile devices' hardware and their cost reduction positions them as the first tool for accessing information worldwide [29]. This trend is consistent with most of the selected studies, which use mobile devices to support AR technology as a tool for improving spatial intelligence.

Before carrying out the different classroom experiences, most authors developed worksheets or augmented books that could be activated through a very heterogeneous selection of software or applications (Table 5). In order to generate this work material, teachers must have sufficient knowledge. In this regard, some authors design their own activity sheets or OER work materials in what they call "production of augmented materials", for which the production process is generally systematic and sequential [2].

\subsection{Limitations of AR in Enhancing Spatial Intelligence (RQ5)}

Having noted the many advantages of AR, several researchers point out that there are certain limitations that this technology could pose in terms of improving spatial skills in STEM subjects (Table 9). These limitations are accentuated by the fact that the applicability of AR in education is still at an early stage and more research is needed (2 of 17).

Table 9. Limitations of AR in improving spatial intelligence.

\begin{tabular}{ccc}
\hline Limitations & $\mathbf{n}_{\mathbf{i}}$ & Sample Research \\
\hline Shortage of learning augmented materials & 6 & Lin et al., 2015 [44] \\
Lack of opportunities for teacher training & 5 & Del Cerro and Morales, 2021 [4] \\
Evaluation tools & 5 & Dünser et al., 2006 [23] \\
Not very effective for students with a high level & 3 & Chen et al., 2011 [7] \\
of spatial skills & 2 & Rohendi et al., 2018 [50] \\
Technology not widely used & 2 &
\end{tabular}

The limitation most reiterated by researchers highlights the scarcity of augmented learning materials available (6 out of 17). In their experience in graphic expression, GómezTone et al. [47] warned that problems may arise when students request more AR learning materials to support the content. However, the lack of resources and the design of augmented materials through SDKs requires a great deal of effort and is geared towards teachers with knowledge in programming and computer-aided design.

The latter is directly related to the lack of teacher training in emerging technologies such as AR ( 5 out of 17), and to the lack of design principles and integration of methodologies through AR [48]. Thus, the need for resources for teachers and the limited opportunities for teacher training limit the incorporation of AR in the classroom [4]. Moreover, as this technology advances, and although SDKs are becoming more direct and intuitive to interface, more training is needed to implement animated representations at a higher graphical resolution [44].

In the third place, future research should focus on a better assessment of spatial intelligence and the effects of AR on long-term learning retention (5 of 17). Some authors propose empirical studies based on how AR can effectively create long-term learning in students [4,45,47]. On the other hand, according to Dünser et al. [23], the measures of spatial ability taken fail to cover all the visuospatial abilities that are used when working in a three-dimensional space, thus calling for new tools to measure spatial intelligence directly in 3D. Gutiérrez et al. [42] propose as a possible solution, the inclusion of virtual self-assessment tests through AR software and applications.

Some researches (3 out of 17) highlighted, that students did not show significant improvements in their spatial skills when they started with a high visuospatial level $[7,44]$. 
Therefore, the effects of AR on the spatial intelligence of these students were lower [49], probably because the range of improvement in their spatial intelligence was smaller than that of students starting from a medium-low level.

\section{Conclusions}

Research reveals that AR technology, accompanied by appropriate methodology, has a positive effect on the development of spatial intelligence of students in STEM subjects. While there has been an increase in studies on educational applications of AR in STEM subjects over the last decade, there is very little formal research focused on the impact of AR on students' spatial intelligence.

In order to obtain information on secondary and higher education students' spatial skills, the authors use standardized tests or psychometrically validated questionnaires as assessment tools, with the most commonly assessed spatial skills being visualization and spatial rotation. On the other hand, this SR revealed that the combination of AR technology and an appropriate methodology in educational environments provides an immersive learning experience for students. There are numerous benefits of using this approach in STEM subject contexts: improved kinesthetic and collaborative learning, visualizations, tangibility with interactive objects, and increased motivation, satisfaction and willingness to learn for students. Likewise, the use of this technology favors digital literacy, a skill that goes beyond the technical expertise to use digital devices and that Zapata-Ros [62] defines as a combination of technical-processual and cognitive skills that are necessary to live, learn and work in a digital society.

When comparing methodologies using AR technology with other types of traditional pedagogical tools in STEM learning with a high proportion of visuospatial content, the results indicate that learning gains are higher when AR resources are used in the interventions. The study assesses students' learning and academic achievements through the grades obtained, and addresses other factors, such as tangibility and motivation, which have positively influenced students' learning outcomes. Therefore, we can affirm that the value of any technology that is integrated in the classroom depends largely on the involvement of students, with AR being a technology that is highly accepted by students. It is important to bear in mind that underdeveloped spatial skills act as a barrier to success in learning STEM subjects [47], so early incorporation of AR in these subjects will facilitate learning throughout the academic years in these areas.

Since 2011, mobile devices have become the most widely used tool for the use of AR to improve spatial intelligence. Specifically, they present a series of pedagogical advantages to which other operational advantages can be added, such as the fact that this tool is available to practically all students from secondary education onwards, and it offers enormous possibilities for interaction in learning environments; it is flexible, small in size, easy to use and its cost can be low in some cases [63]. For all these reasons, it can be stated that the AR-mobile devices binomial is a great support in the process of including AR technology in education, as well as in lifelong learning and multiple contexts, in what today are called learning ecologies. In addition, technological and commercial competition in the mobile device sector is largely supportive of AR technological advances. Likewise, it is necessary to continue research on the AR-mobile devices binomial which, if used appropriately, makes it possible for everyone to improve visuospatial skills and have access to information; thus contributing to achieving sustainable development as promoted by SDG 4 (Quality Education), in which one of its goals focuses precisely on "ensuring that all learners acquire the knowledge and skills needed to promote sustainable development" [29].

We cannot overlook the fact that any technological development "per se" does not represent progress in the development of spatial intelligence or learning. Those involved in the design of these augmented environments must be aware of the technological resources available, as well as their advantages and limitations, in order to be able to relate them to the objectives, contents, strategies, appropriate learning methods and assessment. Therefore, the role of the teacher is of utmost importance in order to guarantee an educational and 
quality use [64]. For this reason, it is necessary to insist on the need for more teacher training opportunities in emerging ICT and the development of more augmented materials in the form of Open Educational Resources (OER) for teachers.

Author Contributions: Conceptualization, F.d.C.V. and G.M.M.; methodology, F.d.C.V. and G.M.M.; software, G.M.M.; validation, F.d.C.V. and G.M.M.; formal analysis, F.d.C.V.; investigation, F.d.C.V. and G.M.M.; data curation, F.d.C.V. and G.M.M.; writing-original draft preparation, F.d.C.V. and G.M.M.; writing-review and editing, F.d.C.V. and G.M.M. All authors have read and agreed to the published version of the manuscript.

Funding: This research received no external funding.

Institutional Review Board Statement: Not applicable.

Informed Consent Statement: Not applicable.

Data Availability Statement: Not applicable.

Conflicts of Interest: The authors declare no conflict of interest.

\section{References}

1. Kaufmann, H.; Schmalstieg, D. Mathematics and Geometry Education with Collaborative Augmented Reality. In Proceedings of the ACM SIGGRAPH, New York, NY, USA, 17-28 August 2002; pp. 37-41.

2. Del Cerro Valázquez, F.; Morales Méndez, G. Realidad Aumentada como herramienta de mejora de la inteligencia espacial en estudiantes de educación secundaria. Rev. Educ. Distancia (RED) 2017, 17, 298831. [CrossRef]

3. Del Cerro Valázquez, F.; Lozano Rivas, F. Proyecto Técnico Ecourbano apoyado en las TIC para el aprendizaje STEM (Dibujo Técnico) y la consolidación de los ODS en el aula. Rev. Educ. Distancia (RED) 2019, 19, 60. [CrossRef]

4. Del Cerro Valázquez, F.; Morales Méndez, G. Application in Augmented Reality for Learning Mathematical Functions: A Study for the Development of Spatial Intelligence in Secondary Education Students. Mathematics 2021, 9, 369. [CrossRef]

5. Maier, P.H. Räumliches Vorstellungsvermögen: Komponenten, Geschlechtsspezifische Differenzen, Relevanz, Entwicklung und Realisierung in der Realschule; Lang: Bern, Switzerland, 1994.

6. Samsudin, K.; Rafi, A.; Hanif, A.S. Training in mental rotation and spatial visualization and its impact on orthographic drawing performance. J. Educ. Technol. Soc. 2011, 14, 179-186.

7. Chen, Y.C.; Chi, H.L.; Hung, W.H.; Kang, S.C. Use of tangible and augmented reality models in engineering graphics courses. J. Prof. Issues Eng. Educ. Pract. 2011, 137, 267-276. [CrossRef]

8. Azuma, R. A Survey of Augmented Reality. Presence Teleoper. Virtual. Environ. 1997, 6, 335-385. [CrossRef]

9. Behzadan, A.H.; Kamat, V.R. Enabling discovery-based learning in construction using telepresent augmented reality. Autom. Constr. 2013, 33, 3-10. [CrossRef]

10. Billinghurst, M.; Duenser, A. Augmented reality in the classroom. Computer 2012, 45, 56-63. [CrossRef]

11. Bacca Acosta, J.L.; Baldiris Navarro, S.M.; Fabregat Gesa, R.; Graf, S. Augmented reality trends in education: A systematic review of research and applications. J. Educ. Technol. Soc. 2014, 17, 133-149.

12. Radu, I. Augmented reality in education: A meta-review and cross-media analysis. Pers. Ubiquitous Comput. 2014, 18, 1533-1543. [CrossRef]

13. Diegmann, P.; Schmidt-Kraepelin, M.; Eynden, S.; Basten, D. Benefits of augmented reality in educational environments-a systematic literature review. Benefits 2015, 3, 1542-1556.

14. Saidin, N.F.; Halim, N.D.A.; Yahaya, N. A review of research on augmented reality in education: Advantages and applications. Int. Educ. Stud. 2015, 8, 1-8. [CrossRef]

15. Cabero Almenara, J.; Barroso Osuna, J.M. Posibilidades educativas de la Realidad Aumentada. J. New Approaches Educ. Res. 2016, $5,46-52$.

16. Hwang, G.J.; Wu, P.H.; Chen, C.C.; Tu, N.T. Effects of an augmented reality-based educational game on students' learning achievements and attitudes in real-world observations. Interact. Learn. Environ. 2016, 24, 1895-1906. [CrossRef]

17. Saltan, F.; Arslan, Ö. The use of augmented reality in formal education: A scoping review. Eurasia J. Math. Sci. Technol. Educ. 2016, 13, 503-520. [CrossRef]

18. Carbonell Carrera, C.; Bermejo Asensio, L.A. Landscape interpretation with augmented reality and maps to improve spatial orientation skill. J. Geogr. High. Educ. 2017, 41, 119-133. [CrossRef]

19. Chen, P.; Liu, X.; Cheng, W.; Huang, R. A review of using Augmented Reality in Education from 2011 to 2016. In Innovations in Smart Learning; Popescu, E., Kinshuk, M.K., Huang, R., Jemni, M., Chen, N.S., Sampson, D.G., Eds.; Springer: Singapore, 2017; pp. 13-18.

20. Dey, A.; Billinghurst, M.; Lindeman, R.W.; Swan, J. A systematic review of 10 years of augmented reality usability studies: 2005 to 2014. Front. Robot. AI 2018, 5, 37. [CrossRef] [PubMed] 
21. López Belmonte, J.; Moreno-Guerrero, A.-J.; López Núñez, J.A.; Pozo Sánchez, S. Analysis of the Productive, Structural, and Dynamic Development of Augmented Reality in Higher Education Research on the Web of Science. Appl. Sci. 2019, 9, 5306. [CrossRef]

22. Shelton, B.E.; Hedley, N.R. Exploring a cognitive basis for learning spatial relationships with augmented reality. Technol. Instr. Cogn. Learn. 2004, 1, 323.

23. Dünser, A.; Steinbügl, K.; Kaufmann, H.; Glück, J. Virtual and augmented reality as spatial ability training tools. In Proceedings of the 7th ACM SIGCHI New Zealand Chapter's International Conference on Computer-Human Interaction: Design Centered HCI, New York, NY, USA, 6 July 2006; pp. 125-132.

24. Thornton, T.; Ernst, J.V.; Clark, A.C. Augmented reality as a visual and spatial learning tool in technology education. Technol. Eng. Teach. 2012, 71, 18-21.

25. Jeřábek, T.; Rambousek, V.; Wildová, R. Specifics of visual perception of the augmented reality in the context of education. Procedia-Soc. Behav. Sci. 2014, 159, 598-604. [CrossRef]

26. Quintero, E.; Salinas, P.; González-Mendívil, E.; Ramírez, H. Augmented reality app for calculus: A proposal for the development of spatial visualization. Procedia Comput. Sci. 2015, 75, 301-305. [CrossRef]

27. Gómez-García, M.; Trujillo-Torres, J.M.; Aznar-Díaz, I.; Cáceres-Reche, M.P. Augment reality and virtual reality for the improvement of spatial competences in Physical Education. J. Hum. Sport Exerc. 2018, 13, 189-198.

28. González, N.A.A. Development of spatial skills with virtual reality and augmented reality. Int. J. Interact. Des. Manuf. (IJIDeM) 2018, 12, 133-144. [CrossRef]

29. Del Cerro Velázquez, F.; Morales Méndez, G. Augmented reality and mobile devices: A binominal methodological resource for inclusive education (SDG 4). An example in secondary education. Sustainability 2018, 10, 3446. [CrossRef]

30. Hinojo Lucena, F.J.; Fernández Martín, F.D.; Díaz Aznar, I. Las actitudes de los docentes hacia la formación en tecnologías de la información y comunicación (TIC) aplicadas a la educación. Contextos Educ. Rev. Educ. 2002, 5, 253-270.

31. Ahmad, N.; Junaini, S. Augmented Reality for Learning Mathematics: A Systematic Literature Review. Int. J. Emerg. Technol. Learn. (iJET) 2020, 15, 106-122. [CrossRef]

32. Herrera, L.M.; Pérez, J.C.; Ordóñez, S.J. Developing spatial mathematical skills through 3D tools: Augmented reality, virtual environments and 3D printing. Int. J. Interact. Des. Manuf. (IJIDeM) 2019, 13, 1385-1399.

33. Akçayır, M.; Akçayır, G. Advantages and challenges associated with augmented reality for education: A systematic review of the literature. Educ. Res. Rev. 2017, 20,1-11. [CrossRef]

34. Gün, E.T.; Atasoy, B. The effects of augmented reality on elementary school students' spatial ability and academic achievement. Egit. Ve Bilim 2017, 42, 191.

35. Kitchenham, B.; Brereton, O.P.; Budgen, D.; Turner, M.; Bailey, J.; Linkman, S. Systematic literature reviews in software engineering-a systematic literature review. Inf. Softw. Technol. 2009, 51, 7-15. [CrossRef]

36. Bar-Ilan, J. Citations to the "Introduction to informetrics" indexed by WOS, Scopus and Google Scholar. Scientometrics 2010, 82, 495-506. [CrossRef]

37. Burnham, J.F. Scopus database: A review. Biomed. Digit Libr. 2006, 3, 1. [CrossRef] [PubMed]

38. Hsu, Y.C.; Hung, J.L.; Ching, Y.H. Trends of educational technology research: More than a decade of international research in six SSCI-indexed refereed journals. Educ. Technol. Res. Dev. 2013, 61, 685-705. [CrossRef]

39. Cohen, J. Weighted kappa: Nominal scale agreement provision for scaled disagreement or partial credit. Psychol. Bull. 1968, 70, 213-220. [CrossRef]

40. Martín-Gutiérrez, J.; Saorín, J.L.; Contero, M.; Alcañiz, M.; Pérez-López, D.C.; Ortega, M. Design and validation of an augmented book for spatial abilities development in engineering students. Comput. Graph. 2010, 34, 77-91. [CrossRef]

41. Contero, M.; Gomis, J.M.; Naya, F.; Albert, F.; Martin-Gutierrez, J. Development of an augmented reality based remedial course to improve the spatial ability of engineering students. In Proceedings of the 2012 Frontiers in Education Conference Proceedings, Seattle, WA, USA, 3-6 October 2012; pp. 1-5.

42. Gutiérrez de Ravé, E.; Jiménez-Hornero, F.J.; Ariza-Villaverde, A.B.; Taguas-Ruiz, J. DiedricAR: A mobile augmented reality system designed for the ubiquitous descriptive geometry learning. Multimed. Tools Appl. 2016, 75, 9641-9663. [CrossRef]

43. Liao, Y.T.; Yu, C.H.; Wu, C.C. Learning Geometry with Augmented Reality to Enhance Spatial Ability. In Proceedings of the 2015 International Conference on Learning and Teaching in Computing and Engineering, Taipei, Taiwan, 9-12 April 2015.

44. Lin, H.C.K.; Chen, M.C.; Chang, C.K. Assessing the effectiveness of learning solid geometry by using an augmented realityassisted learning system. Interact. Learn. Environ. 2015, 23, 799-810. [CrossRef]

45. Bogomolova, K.; van der Ham, I.J.; Dankbaar, M.E.; van den Broek, W.W.; Hovius, S.E.; van der Hage, J.A.; Hierck, B.P. The effect of stereoscopic augmented reality visualization on learning anatomy and the modifying effect of visual-spatial abilities: A double-center randomized controlled trial. Anat. Sci. Educ. 2020, 13, 558-567. [CrossRef] [PubMed]

46. Camba, J.; Contero, M.; Salvador-Herranz, G. Desktop vs. Mobile: A Comparative Study of Augmented Reality Systems for Engineering Visualizations in Education. In Proceedings of the 2014 IEEE Frontiers in Education Conference (FIE) Proceedings, Madrid, Spain, 22-25 October 2014; pp. 1-8.

47. Gómez-Tone, H.C.; Martin-Gutierrez, J.; Valencia Anci, L.; Mora Luis, C.E. International comparative pilot study of spatial skill development in engineering students through autonomous augmented reality-based training. Symmetry 2020, $12,1401$. [CrossRef] 
48. Habig, S. Who can benefit from augmented reality in chemistry? Sex differences in solving stereochemistry problems using augmented reality. Br. J. Educ. Technol. 2020, 51, 629-644. [CrossRef]

49. Omar, M.; Ali, D.; Mokhtar, M.; Zaid, N.; Jambari, H.; Ibrahim, N. Effects of Mobile Augmented Reality (MAR) towards students' visualization skills when learning orthographic projection. Int. J. Emerg. Technol. Learn. (iJET) 2019, 14, 106-119. [CrossRef]

50. Rohendi, D.; Septian, S.; Sutarno, H. The Use of Geometry Learning Media Based on Augmented Reality for Junior High School Students. In IOP Conference Series: Materials Science and Engineering; IOP Publishing: Manado, Indonesia, 2018; Volume 306, p. 12029.

51. Roca González, C.; Martín Gutierrez, J.; García Dominguez, M.; Mato Carrodeguas, M.D.C. Virtual Technologies to Develop Visual-Spatial Ability in Engineering Students. Eurasia J. Math. Sci. Technol. Educ. 2017, 13, 441-468. [CrossRef]

52. Linn, M.C.; Petersen, A.C. Emergence and Characterization of Sex Differences in Spatial Ability: A Meta-Analysis. Child Dev. 1985, 56, 1479-1498. [CrossRef] [PubMed]

53. Gutierrez, A. Visualization in 3-Dimensional Geometry: In Search of a Framework. In Proceedings of the 20th International Conference for the Psychology of Mathematics Education, Valencia, Spain, 8-12 July 1996.

54. Blanco, T.; Pegito, J.; Godino, J. Configuraciones Epistémicas y Cognitivas en Tareas de Visualización y Razonamiento Espacial; Investigación en Educación Matemática: Madrid, Spain, 2008; pp. 189-198. ISBN 978-84-612-5856-7.

55. Shepard, R.N.; Metzler, J. Mental rotation of three-dimensional objects. Science 1971, 171, 701-703. [CrossRef] [PubMed]

56. Vandenberg, S.G.; Kuse, A.R. Mental rotations, a group test of three-dimensional spatial visualization. Percept. Mot. Ski. 1978, 47, 599-604. [CrossRef]

57. Navarro, R.; Saorín, J.; Martín-Dorta, N.; Martín Gutiérrez, J. Expresión Gráfica en Arquitectura e Ingeniería y el Desarrollo de la Visión Espacial y Habilidades Espaciales de los Alumnos de las Carreras Técnicas. In Proceedings of the VIII Congreso de Expresión Gráfica Aplicada a la Edificación, Madrid, Spain, 27-28 May 2006.

58. Bennett, G.K.; Seashore, H.G.; Wesman, A.G. Differential Aptitude Test: Forms S and T; The Psychological Corporation: New York, NY, USA, 1973.

59. Lieu, D.K.; Sorby, S.A. Visualization, Modeling, and Graphics for Engineering Design; Delmar, Cengage Learning: Clifton Park, NY, USA, 2009.

60. Guay, R. Purdue Spatial Visualization Test; Purdue University: West Layfette, IN, USA, 1977.

61. Sorby, S.A. Developing 3D spatial skills for engineering students. Australas. J. Eng. Educ. 2007, 13, 1-11. [CrossRef]

62. Zapata-Ros, M. Pensamiento computacional: Una nueva alfabetización digital. Rev. De Educ. A Distancia (RED) 2015, 46, 1-47. [CrossRef]

63. Rivera Alvarado, L.A.; López Domínguez, E.; Hernández Velázquez, Y.; Domínguez Isidro, S.; Excelente Toledo, C.B. Layered Software Architecture for the Development of Mobile Learning Objects with Augmented Reality. IEEE Access 2018, 6, 57897-57909. [CrossRef]

64. Cabero Almenara, J.; Llorente Cejudo, M.D.C.; Román Graván, P. Las herramientas de comunicación en el" aprendizaje mezclado". Pixel-Bit. Rev. Medios Y Educ. 2004, 23, 27-41. 